\title{
Application and Development Trend of Automotive Electronic Technology
}

\author{
Zhixin Wang \\ Huizhou Economics And Polytechnic College, Huizhou, Guangdong 516057, China
}

Keywords: automotive electronic technology; application development; electric power control system; computer

\begin{abstract}
Automotive electronics technology is a subject that uses power electronic devices to control and convert automotive electronic devices. The generation link of automotive electronic control system designs a variety of equipment for generating units. According to the development of automotive electronic technology, the improvement analysis of automotive electronic technology is carried out, and the development of automotive electronic technology is analyzed in combination with the application status of engine, body and chassis, as well as in automotive safety system. According to the engine load speed, brake working state and all kinds of parameters controlled by the driver, the electronic control device of the transmission system can automatically adapt to the change of instantaneous working condition. With the continuous development of information technology to the depth and breadth, the future automobile will inevitably be "intelligent vehicle" which integrates the latest technologies such as electronics, computer, communication technology and so on, so as to improve the intelligent control ability of automobile electronics.
\end{abstract}

\section{Introduction}

In recent years, with the rapid development of automobile industry and transportation industry in China, automobile has become an indispensable vehicle for people to work, study and live. With the rapid development of automobile industry and the increasing quantity of automobile market, higher requirements are put forward for automotive electronic control technology. With the development of detection and diagnosis technology, it plays a very important role in the search of vehicle running status and fault location ${ }^{[1]}$. Intelligence lays an important technical foundation, and it also depicts a broad prospect for the continuous expansion and innovation of automotive electronics technology in the 21st century. Compared with the developed countries, there is still a big gap in the comprehensive technical ability of developing and developing automotive electronic devices in China. In order to promote the technological innovation of automobile electronic device manufacturing process and improve the reliability of the device, it is necessary to guide innovation in device manufacturing technology and to innovate in the application of new material science.

\section{Composition of vehicle electronic technology and the application of automobile information technology.}

The application of automobile information technology is based on the GIS, mobile communication network of global positioning system (GPS,) geographic information system (GIS,) and the international network transmission control (TCP/IP) technology ${ }^{[2]}$. It can easily realize data transmission, voice communication and target tracking in automobiles. Automatic 
alarm, as well as all kinds of public information, practical information service function, and it can be combined with 110120 and other systems and various databases to achieve a wider application.

\subsection{Adaptive cruise control system}

The adaptive cruise control system uses radar, sonar and laser beams to scan the road ahead by controlling the vehicle and setting the desired lower speed. When necessary, the adaptive cruise control system will automatically reduce valve opening, gear reduction and even braking to maintain a safe distance. But this system requires special sensors on the road and on the car! Control and communication equipment, can achieve unmanned driving. These cars are generally equipped with precise positioning systems that facilitate travel for the elderly, infirm and disabled.

\subsection{Integrated security system}

Integrated security system is composed of dozens of technologies, including electronic devices, microcontrollers, sensors and other technologies and products. The integrated safety system can be developed with advanced electronic technology and integrated technology. The anti-collision warning and collision notification system also uses radar sonar and laser beam scanning to scan potential hazards and obstacles and send out emergency signals in case of an imminent car collision or traffic accident in order to avoid an accident.

\subsection{Network communication and electronic navigation system}

The network communication system allows the driver to receive network news, email and other information through a portable computer and cordless phone, with eyes on the way forward and hands on the steering wheel. And can be transmitted to the driver through the voice control voice system.

Electronic navigation system is also called vehicle navigation system. It uses the navigation function of global positioning system (GPS) to help drivers choose the best route in the complex urban road traffic network and make it reach its destination quickly.

\section{Application of automotive electronic technology in electric vehicle driving system.}

Automotive electronic drive system is mainly composed of motor, power conversion and control technology. ESA is composed of computer, sensor and its interface, actuator and so on ${ }^{[3]}$. The device can be calculated and judged according to the engine parameters sent by the sensor, and then the ignition time can be adjusted to save fuel and reduce air pollution.

The application of automotive electronic technology is mainly reflected in the following aspects:

\subsection{Application in engine}

The application of electronic technology in the engine can achieve low fuel consumption and low pollution, reduce the impact of power transmission system, and improve the power performance and economy of the automobile. The engine can be operated under the optimal working conditions, and the comprehensive performance of the engine can be improved. The structure of the automotive electronic system is shown in figure 1. 


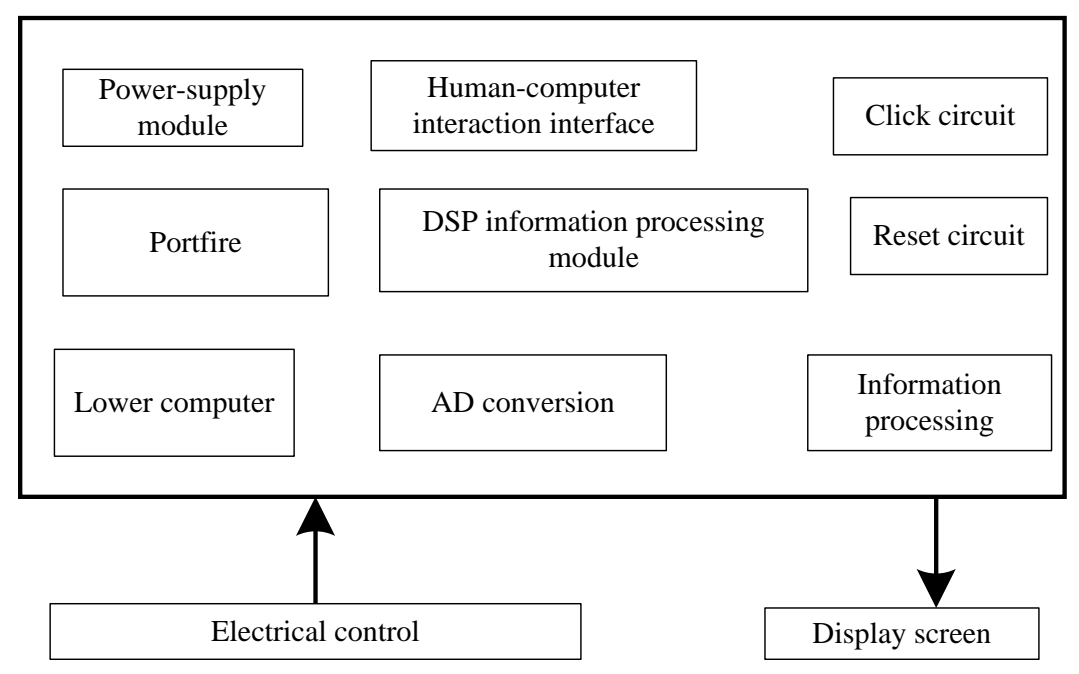

Figure 1 composition of automotive electronic system

\subsection{Electronic control automatic transmission (ECAT)}

ECAT can be based on engine load speed, The speed, the working state of the brake and the various parameters controlled by the driver are automatically changed after the calculation of the computer, and the position of the gearshift is automatically changed, so as to realize the best control of the gear shift of the transmission ${ }^{[4]}$. It has the advantages of good acceleration performance, high sensitivity, and can accurately reflect vehicle driving load and road conditions. The shift control can realize the optimal economy and comfort of the vehicle.

\subsection{Anti-lock braking system (ABS)}

The system is an important security component which has the longest development time and the most rapid application in automobile electronic device. It ensures the optimum slip rate between the wheels and the ground by controlling to prevent the wheels from clinging to the ground during braking, thus enabling the car to brake on all kinds of roads. Reducing braking distance driving anti-slip system (ASR) is also called traction control system. It is the perfect and supplement of anti-lock braking system. It can prevent the driving wheel skidding when the vehicle starts and accelerates, and it can help to improve the traction performance of the vehicle. It can also improve its operation stability.

\subsection{Adaptive suspension system (ABC)}

The adaptive air suspension system can automatically adjust the stiffness of the suspension air spring and the damping characteristics of the shock absorber according to the instantaneous load of the suspension device in order to adapt to the current load and maintain the fixed height of the suspension. Making cars run more smoothly, while ensuring effective control of body height at any speed, and further improving the interconnections between suspension devices, which can greatly improve the stability of the vehicle, Maneuverability, possibility and ride comfort.

\subsection{Active cruise automatic control system (ACC)}

Active cruise automatic control system includes radar sensor, digital signal processor and control module. When a vehicle travels at a high speed and long distance, it can automatically control the brake and speed to ensure a certain distance. Avoid frequent cancellation and setup of cruise control, creating a more relaxed driving atmosphere. 


\section{Development direction of automobile electronic control technology.}

\subsection{Basis of automotive electronics technology standardization}

With the continuous development of automobile industry and transportation industry, and the continuous improvement of automotive electronic control technology, we should focus on the standardization of the basis of automobile detection technology in the future, and further improve the testing technology software matching with hardware. For example, to formulate and perfect the test method and limit value standard of the automobile test project, to formulate the detailed rules for the inspection and evaluation of the technical condition of the operating vehicle, and to unify and standardize the testing requirements ${ }^{[5]}$. Therefore, in order to improve the comprehensive performance of the automobile and prolong the service life of the automobile, our country should also develop towards the direction of monitoring and predicting the technical situation of the automobile at present.

\subsection{Application of photoelectric technology and computer processing technology.}

At present, a large number of foreign automobile inspection equipment have applied the integrated technology of light, machine and electricity, as well as the computer measurement and control, which can automatically identify and detect the status of the automobile technology, and can diagnose the position and cause of the automobile malfunction ${ }^{[6]}$. Therefore, our country should apply the photoelectric technology and the computer processing technology to the automobile electronic control technology as soon as possible.

\subsection{Intelligent automobile electronic equipment}

Some automobile inspection equipment abroad have the function of expert system and intelligence, but at present, there is still a big gap in the use of expert system and intelligent diagnosis between China's automobile inspection equipment and foreign countries. Therefore, in the future, we will speed up the development of intelligent automobile testing equipment.

\section{Application of automotive electronic technology}

\subsection{Overall design of automotive electronic control system.}

To realize the optimal design of automobile electronic system, the whole frame of automobile electronic system is described firstly, and the principle of automobile electronic control is analyzed. Taking Linux as the embedded kernel of microcomputer, the development platform of automobile electronic system is constructed, and the characteristic information of electromagnetic compatibility signal of city subway is processed by adaptive detection. The bus transmission design of automobile electronic system is carried out by using ISA/EISA/Micro Channel extended bus, and the output current equalization and adaptive control of the electromagnetic sensor of urban subway is carried out by using frequency domain equalization control method. The communication design of upper computer of automobile electronic system is carried out in DSP. Adopting embedded IVI (Interchangeable Virtual Instruments, interchangeable virtual instrument) as the main control chip of automobile electronic control system, the main control module of automobile electronic control is designed. Thus, the overall framework of the automotive electronic system designed in this paper is shown in figure 2. 


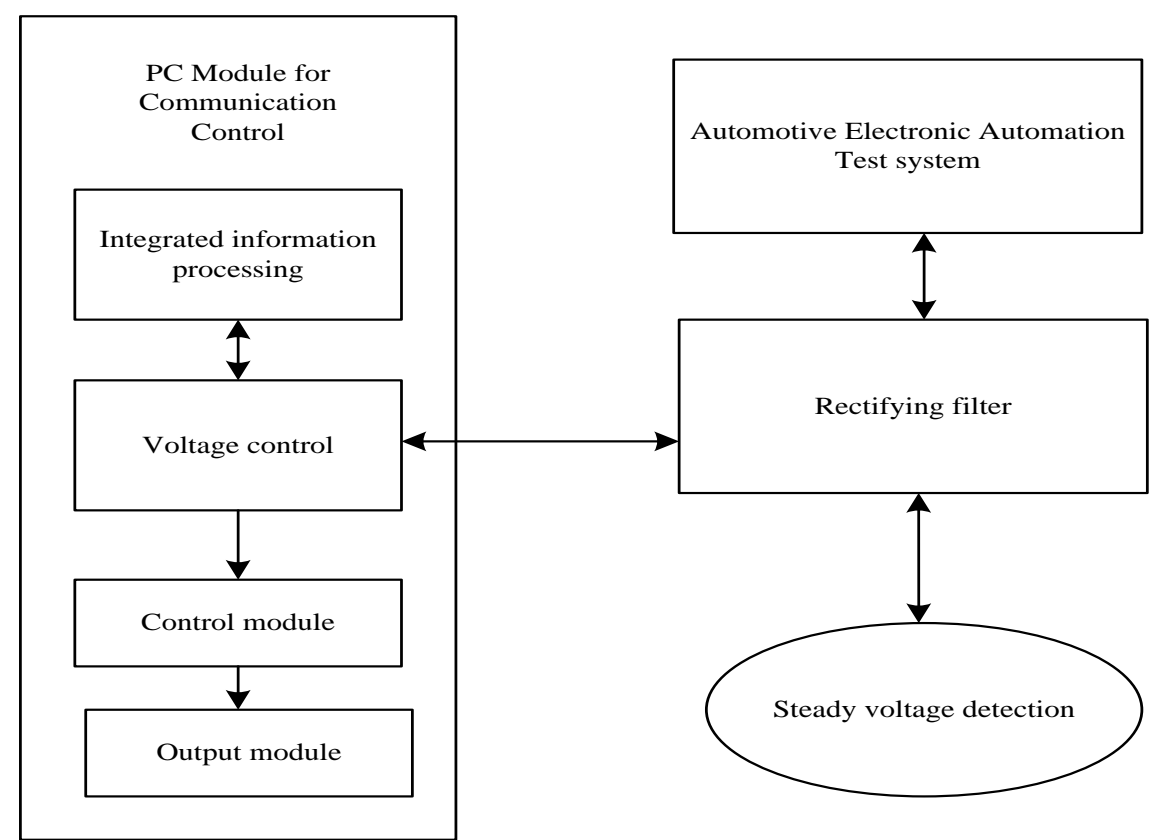

Figure 2 Overall design framework of the system

\subsection{System test and experimental analysis.}

Aiming to verify the application performance of this method in the automatic test of electronic compatibility of metro vehicle, the system is debugged and analyzed, the port is initialized, and the sleep time (1s) is set. According to the state of the control word equipment of the automatic test system, the control network module of the automobile electronic system is designed by using the flexible networking mode of ARM in the Internet of things environment. The signal source produces four different waveforms of the 1KHz. 256 sampling points are set at the A/D end of the automotive electronic compatibility test system, and the acquisition parameters are repeatedly collected to realize the compatibility test of automobile electronics. The test results are shown in figure 3 . The test results show that the system has good stability and good compatibility.
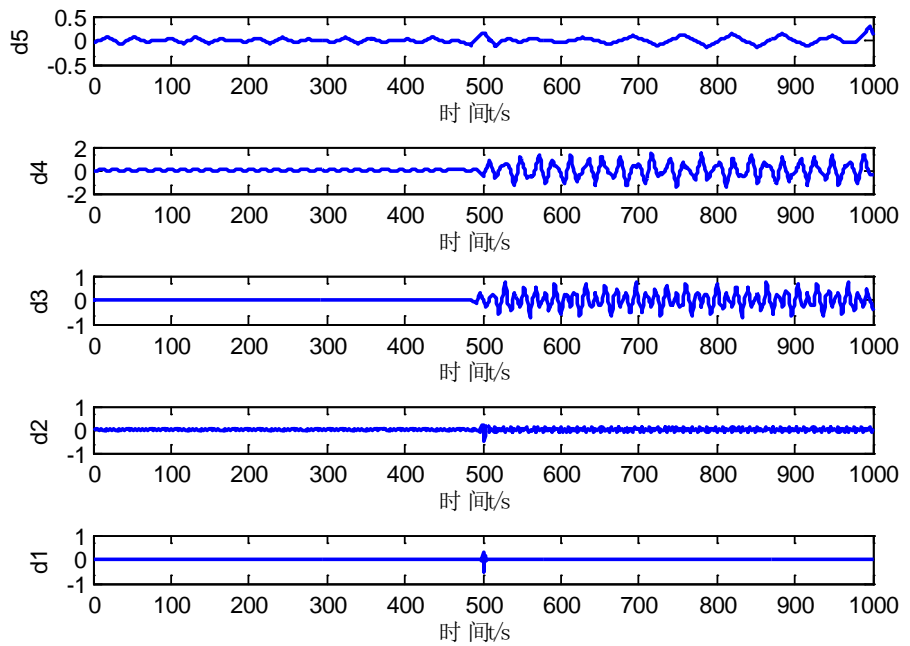

Figure 3 Automotive electronic test results

\section{Conclusions}

According to the development of automotive electronic technology, the improvement analysis 
of automotive electronic technology is carried out, and the development of automotive electronic technology is analyzed in combination with the application status of engine, body and chassis, as well as in automotive safety system. According to the engine load speed, brake working state and all kinds of parameters controlled by the driver, the electronic control device of the transmission system can automatically adapt to the change of instantaneous working condition. Keep the engines working at the lowest possible speed. The stepless automatic transmission is a metal belt (chain) in the middle of a variable diameter drive wheel to transfer torque and continuously change the speed ratio. Electronic technology is widely used in automobile technology so that the control of the car is rapidly electrified. With the continuous development of information technology to the depth and breadth, the future automobile is bound to be a "smart car" that integrates the latest technologies, such as electronics, computers, communications technology, etc. In order to improve the intelligent control ability of automobile electronics and increase the investment in automotive electronic control technology, the automobile detection technology can develop rapidly and make due contribution to the economic development of our country.

\section{References}

[1] JU C H, ZOU J B. An incremental classification algorithm for data stream based on information entropy diversity measure[J].Telecommunications Science, 2015, 31(2):86-96.

[2] BAO J, ZHENG Y, MOKBEL M F. Location-based and preference-aware recommendation using sparse geo-social networking data[C]//Proceedings of the 20th International Conference on Advances in Geographic Information Systems. New York:ACM, 2012:199-208.

[3] JU C H, ZOU J B. An incremental classification algorithm for data stream based on information entropy diversity measure[J].Telecommunications Science, 2015, 31(2):86-96.

[4] LYU Y X, WANG C Y, WANG C, et al. Online classification algorithm for uncertain data stream in big data[J].Journal of Northeastern University (Natural Science Edition), 2016, 37(9):1245-1249.

[5] CHEN Y, LI L J. Very fast decision tree classification algorithm based on red-black tree for data stream with continuous attributes[J]. Journal of Nanjing University of Posts and Telecommunications (Natural Science Edition), 2017, 37(2):86-90.

[6] QIAO Z W, SUN W X. Two improments to C4.5 algorithm[J]. Journal of Jiangsu Polytechnic University, 2008, 20(4):56-59. 